$\underline{\xi}=$ 离

\title{
CNS tuberculosis: diagnostic challenges and review of recent laboratory investigations
}

\author{
Vinod K.S. Gautam ${ }^{1 *}$, Renu Gupta ${ }^{2}$ \\ ${ }^{1}$ Assistant Professor, Department of Neurosurgery, Institute of Human Behaviour and Allied Sciences, \\ Dilshad Garden,Delhi-110095, India \\ ${ }^{2}$ Assistant Professor, Department of Microbiology, Institute of Human Behaviour and Allied Sciences, \\ Dilshad Garden, Delhi-110095, India \\ *Corresponding author E-mail:drvksg@gmail.com
}

\begin{abstract}
Tuberculosis of the central nervous system poses a diagnostic and therapeutic challenge to the physicians. Early diagnosis is warranted to reduce morbidity and mortality associated with this disease. Microbiological investigations for the diagnosis of tuberculosis of central nervous system are of paramount significance. However, due to relative inaccessibility of approaching infected lesions in eloquent area of brain and difficulty in retrieving pathological sample from deep located regions of the brain and spinal cord without causing any neurological deficit, there is need to review the relevance of available microbiological and biochemical tests. Some tests which may be very specific like AFB microscopy and culture may not be positive in many cases due to paucibacillary CSF sample or pus or granulation tissue from the brain or spine. So, authors have reviewed different biochemical and microbiological tests and suggested a pragmatic and step wise practical approach for use of laboratory investigations in clinical management of CNS TB patients.
\end{abstract}

\section{Introduction}

Tuberculosis (TB) is caused by Mycobacterium tuberculosis, an acid fast bacillus (AFB) and obligate aerobe. Central Nervous System Tuberculosis (CNS TB) is extrapulmonary manifestation of Tuberculosis. Early diagnosis and treatment arenecessary to reduce the neurological morbidity associated with CNS Tuberculosis. Various investigations are used to diagnose CNS TB, however, the diagnosis of CNS TB is often delayed because of the subtle and non specific clinical presentations of the patients. Moreover, it is relatively difficult to access the pathological site and collect infected material from eloquent areas of CNS. Therefore, the diagnosis of CNS TB is very often challenging. So, clinical observation, neuro-radiological investigations and application of relevant biochemical and microbiological tests are needed to start patient specific anti tubercular therapy to reduce high morbidity and mortality associated with this disease.

\section{Relevance of pathological sample in neuro- tuberculosis}

All the patients suspected to have CNS TB should be investigated systematically through various diagnostic tests. Blood, sputum, CSF or granulation tissue are subjected to variousbiochemical and molecular investigations to find out any evidence of tuberculous infection [Gautam et al. 2013].

So, an attempt should always be made to obtain a pathological sample at the start of the anti tuberculous therapy (ATT).Once sample is obtained in cases of CNS TB , there is a strong possibility that it will be the first and last sample in that particular patient because of the relative inaccessibility of the deeply located lesions and lesions in eloquent area of the brain and spine.So, the advanced technologies like ultrasound or CT guided biopsy or stereotactic biopsy may be required in certain cases of CNS TB. And, once sample is obtained all possible tuberculosis tests like cytology and biochemistry, AFB stain,culture and sensitivity, Nucleic acid amplification test ( NAAT), PCR,molecular test and drug sensitivity testing should be used in each case depending upon available resources.

\section{Use of hematological tests}

If pathological tissue or specimen could not be obtained or the examination of the pathological specimen is not conclusive then other investigations which may support the diagnosis should be analyzed. Blood profile study includes complete blood count ( $\mathrm{CBC}$ ), Erythrocyte Sedimentation Rate (ESR), Liver function Test (LFT)and some other studies depending upon clinical presentation of the patient andresults of other investigations.

Hematological investigations may be perceived as a routine but sometimes these investigation results are very valuable to guide the treatment and follow up.

Anemia may be either pre existing or may develop during the course of treatment.So, hemogram including hematocrit, mean corpuscular volume (MCV) and peripheral blood smear (PBS)mayindicate the type of anemia.Hepatotoxicity is a very common side effect of ATT, so a baseline liver function test (LFT) must be done at the time of initiation of the treatment. Hyperbili- 
rubinemia or any significant change in liver enzymes like SGOPT, SGPT, Alkaline phosphatase warrants the change of ATT regimen. Erythrocyte sedimentation rate (ESR) is a reliable marker for the follow up of the patient.

\section{Need to find any evidence of TB in the body}

Chest radiography, Tuberculin skin testing (Montoux test) and two sputum smears for acid fast bacilli (AFB) should be done for each patient.An attempt should be made to get sputum examination results even in patients with extrapulmonary tubercular involvement. Sputum examination for AFB microscopy and AFB culture may reveal Mycobacteria.Malik et al reported sputum positivity in 6.6 percent of his study subjects with CNS tuberculosis [Malik et al, 2002].

All patients should be examined for any lymhadenopathy. Soft to firm, multiple, matted, enlargedlymph nodes may show the evidence of TB infection. Fine needle aspiration cytology (FNAC) of the palpable lymph node should be done and the material may also be sent for PCR. If it is inconclusive, the excisional biopsy and histopathological examination of the easily accessible lymph node like cervical lymph node should be done.

Similarly, a patient of CNS TB may have concomitant aortic lymhadenopathy or ascites or prevertebral granulomatous or pus collection in the abdomen, USG abdomen may also help in establishing the diagnosis. CT scan of the chest or abdomen may be required if the chest roentgenogram or USG abdomen is suggestive of tuberculous infection and there is need to rule out other diseases like cancers. PET CT or MRI may be required in certain cases to differentiate tuberculosis from malignancies.

\section{Use of biochemical, microbiological and pathological tests}

\subsection{CSF Specimen collection}

If a patient presents with features of tuberculous meningitis and CT scan or MRI of the brain shows hydrocephalus, then ventricular tapping or lumbar Puncture is to be done to collect CSF.

The rationale for doing ventricular tap is to avoid tentorial or tonsillar herniation in patients of TBM with large tuberculoma or tubercular abscess with edema and raised ICT. Ventricular tap is easy in infants and children of less than 18 months where anterior fontanelle is open. But in adultsventricular tap requiresdrilling of bone with twist drill which can be done under local anesthesia. A small twist drill is made just anterior to the coronal suture about 3 $\mathrm{cm}$ lateral to the midline followed by introduction of ventricular tap needle in the right frontal horn of lateral ventricle. This procedure can be performed at the bed side or in the ICU aseptic measures. Ventricular tap may be a life saving procedure in patients of TBM patients presenting with acute hydrocephalus. All centers treating patients of CNS TB cases should have facility for ventricular tapping. If CSF is turbid and has biochemistry and microscopy suggestive of pyogenic meningitis, then external ventricular drainage should be done. Moreover, ventricular CSF could also be collected at the time of insertion of ventricular end during ventriculo-peritoneal shunt surgery.

CSF is collected in a sterile container under aseptic conditions. Volume of CSF plays a critical role in diagnosing CNS tuberculosis and 5- $10 \mathrm{ml}$ of CSF should be collected as sensitivity of diagnosis increases with increase in volume of CSF being processed. Several case series also established CSF culture sensitivities of 25 to $70 \%$ [Naughten et al. 1981, Kent 1993]. Samples from cisternal and ventricular CSF appear to have a higher culture sensitivity than conventional (lumbar puncture) CSF samples [Verdon et al.1996]. A recent study established that both CSF volume and duration of the microscopic evaluation are independently associated with bacteriological confirmation of CNS tuberculosis, suggest- ing that a minimum of $6 \mathrm{ml}$ of CSF fluid should be examined microscopically for a period of $30 \mathrm{~min}$ [Thwaites et al. 2004].

The value of collected CSF in management of CNS TB is immense. Cerebrospinal fluid (CSF) examination should comprise of the entire battery of Biochemistry (proteins, sugars), Cytology, Gram stain, AFB smear, India ink stain, PCR and Culture should be done for all the patients. So, at this stage many other diseases which are considered as differential diagnosis of CNS TB may be ruled out. Pyogenic, Cryptococcal and viral meningitis may be ruled out by CSF examination alone [Thwaites et al. 2004].

\subsection{Cerebrospinal fluid analysis}

CSF in TBM mostly has a clear appearance but often develops fibrin web when allowed to stand. There is pleocytosis, i.e, increased cells in the CSF (10-500 cells/ul) with lymphocytic predominance $(>50 \%)$, elevated protein concentration $(>100 \mathrm{mg} / \mathrm{dl})$ and CSF to plasma glucose ratio of less than $50 \%$ or an absolute CSF glucose concentration of less than $40 \mathrm{mg} / \mathrm{dl}$ [Jeren\&Beus 1982, Verdonet a.1996, Sutlas et al 2003].

\subsection{Microscopy}

Detection of AFB in CSF samples using ZiehlNeelson method of staining is still the most widely accepted technique for diagnosing TB especially in resource limited settings. CSF should be processed in a microbiology laboratory within 1 hour after collection in 3 tubes

(atleast $1 \mathrm{ml}$ in each) for microbiology, cytology and biochemistry [CDC, 2009].

Standard staining techniques using such stains as Ziehl-Neelsen, Kinyoun, or auramine-rhodamine applied to CSF samples have been estimated to detect approximately $100 \mathrm{AFB} / \mathrm{ml}$ of CSF. It is rapid, easy to perform technique but in paucibacillary diseasesensitivity of this test is compromised [Verdon et al, 1996, Cherian\& Thomas, 2011]. The sensitivity of smear microscopy generally varies from $0-40 \%$ with very few studies reporting a very high sensitivity of up to $87 \%$. This high sensitivity has been largely irreproducible in most of the routine diagnostic laboratories. In clinical study the sensitivity of smear microscopy on $\mathrm{ZN}$ staining has been of the order of 5-6\% only.

The sensitivity of microscopy is influenced by large number of factors like clinical case definition used for diagnosis, the volume of CSF processed and the skill of the technician examining the slide. The sensitivity of smear microscopy in TBM can be increased by examination of the spun deposit of large volume CSF samples ( $>6 \mathrm{ml})$, several CSF specimens collected over a few days and prolonged slide examination $(30 \mathrm{~min})$ but these are rarely achieved in clinical practice. Pre treatment of CSF with Triton or cytospin prior to $\mathrm{ZN}$ staining has also been recently shown to increase sensitivity of smear microscopy.

The advances in form of fluorescent microscopy and lightemitting diode (LED) fluorescent microscopy has increased the sensitivity of smear microscopy but the equipments requiredfor these are not readily available in routine laboratories.

\subsection{Culture of Mycobacterium from CSF}

Identification of AFB in the CSF through both smear and culture methods remains the most important and most certain and reliablemeans to diagnose CNS tuberculosis. Growth of Mycobacteria on culture allows drug sensitivity testing, which guides appropriate drug selection. So, traditionally, AFB culture and sensitivity is considered the gold standard investigation for diagnosis of CNS tuberculosis.

Despite its importance among the diagnostic methods used for CNS tuberculosis, traditional staining and culture remain relatively insensitive, most likely due to the typical paucity of AFB in a clinical case of CNS tuberculosis [Verdon et al. 1996].

Culture of M. tuberculosis from CSF is still considered the gold standard for diagnosing TBM and is important for phenotypic 
drug susceptibility testing (DST) and confirmation of drug susceptibility results obtained by other rapid molecular techniques.

M. tuberculosis can be cultured on egg based solid media or on liquid medium but it requiresapproximately 100 bacilli/ $\mathrm{ml}$ and prolonged incubation varying from 6-8 weeks for the culture to be positive. The sensitivity of culture varies from and $20-70 \%$ depending upon case definition used, type of culture media used (solid/ broth based/automated) and volume of CSF inoculated in culture.

The broth based methods (liquid culture media) have shown an increased sensitivity of detecting M. tuberculosis but both liquid and solid media should be used for optimum detection of tubercle bacilli as sometimes $M$. tuberculosis fails to grow on one of the media.

The advent of automated culture techniques like BACTEC MGIT 960, BACT Alert 3D have increased the sensitivity of detecting M. tuberculosis along with decrease in turnaround time to 3-6 weeks but cultures must be incubated up to 8 weeks before considering it as negative.

BACTEC is a rapid culturing method but expensive. Bactec radiometric culture system which can detect mycobacterium as early as 7-14 days on the basis of release of radiolabelled $\mathrm{CO} 2$ from growth of Mycobacteria is selective liquid media using C14 labelled substrate with sensitivity of almost $95 \%$. Bactecsystem is a good system for the diagnosis of extrapulmonary and smear negative tuberculosis.

After isolation, confirmation of isolate as M. tuberculosis and drug susceptibility testing takes another 2-3 weeks by phenotypic culture method.

\subsection{Sensitivity tests}

In this a number of culture media containing serial concentrations of the ATT are inoculated and minimum inhibitory concentrations calculated from the medium with least drug concentration that inhibits growth. Similarly various drugs such as Isoniazid, Rifampicin, etc are placed on culture media and inoculated with concentrated sample of patient's pathological sample. If growth appears in any of the culture media this indicates thatbacillus is resistant to that drug and where growth does not appear, the drug can successfully prevent the growth and bacilli are sensitive to that drug.

\subsection{Microscopic observation drug susceptibility (MODS) assay}

This assay is a simple and inexpensive alternative for detection of M. tuberculosis and DST in liquid culture media using an inverted microscope. In this specimens are inoculated into broth, with and without antibiotics and are examined frequently after 5-7 days incubation for the presence of cording in control tubes. This assay has been found to be more sensitive than smear microscopy and compares favorably with conventional culture methods for diagnosing TBM with a significantly shorter detection time (median 6 days). The performance of this assay for DST has not been evaluated in CSF

\section{New microbiological tests}

Results of classical microbiological techniques traditionally used in the diagnosis of TBM are often delayed and suboptimal. Rapid and accurate diagnostic methods are necessary to facilitate early treatment.As Z-N stain method of bacteriological examination of CSF is only about $25 \%$ sensitive and culture, although $100 \%$ specific, is only $18-83 \%$ sensitive. Moreover, the CSF should be examined before or just after the start of ATT. So, attention has focused on the development of alternative, rapid, accurate methods for the detection of $\mathrm{M}$. tuberculosis in the CSF. Both indirect and direct assays have been evaluated [Thwaites GE et al, 2004]

\section{Direct assays}

PCR test comprises of amplification of target nucleic acid enzymatically by polymerase chain reaction and then using nucleic acid probes for specific detection and identification. The test may detect positive signals from 1-10 bacilli in specimen tested, within a day. The test requires high level of standardization.

Direct assays include Nucleic acid amplification tests (NAAT). Molecular diagnostic methods based on nucleic acid amplification (NAA) techniques allow detection of $\mathrm{M}$. tuberculosis specific nucleotides and are emerging as promising technologies for rapid diagnosis of TBM. The DNA PCRs can detect MTB DNA even after 1 month of start of ATT as these assays cannot differentiate viable and nonviable organisms whereas mRNA based reverse transcriptase PCR can rapidly differentiate viable and non viable M. tuberculosis because of short half-life of bacterial mRNA.

The NAATs involves three main steps: nucleic acid extraction (DNA) from clinical sample, amplification of DNA and detection of amplified product. Ultimate success of these tests depends upon efficient extraction of nucleic acid from clinical sample and good amplification and detection technology. Fundamentally, the sensitivity of detecting $\mathrm{M}$. tuberculosis depends upon the burden of organisms and thereby the target DNA present in clinical specimen and these tests perform better with the larger burden of microorganism. Though NAATs offer a faster diagnosis leading to early initiation of therapy but are highly challenging to optimise in CSF samples due to very small number of bacilli in CSF and presence of amplification inhibitors.

There are numerous detectable gene targets present in MTB genome and multiple types of in house and commercial NAA assays available for detection of TB bacilli with their own merits and demerits.Real-time PCR based assay are associated with decreased chances of contamination and enables quantification of bacterial load with shortened turnaround time whereas multiplex PCR allow for amplification of two or more gene targets simultaneously thus increasing the sensitivity. Nested PCR are two step amplification procedures in which first PCR is followed by an additional amplification with a second set of primers which are complimentary to sequences internal to the sequence targeted by first set of primers and offers increased sensitivity.

\subsection{In-house assay}

A large number of gene targets like IS6110, TRC 4, dev R present in $\mathrm{M}$. tuberculosis genome have been evaluated by various studies for diagnosing TBM. These tests have a higher specificity but a highly variable sensitivity varying from $40-94 \%$ and may show positive results in PCR negative CNS TB cases.

Out of all the gene targets, IS 6110 has been extensively evaluated and found to be more sensitive than other gene targets probably because of presence of multiple copies of IS 6110 in M. tuberculosis genome. However, in house tests are difficult to standardise, requires very good laboratory infrastructure, trained technical staff and strict quality control to prevent cross contamination, detect inhibition and monitor assay performance.

\subsection{Commercial assays}

A large number of commercial assays based on different gene targets and PCR principles are approved by the US Food and Drug Administration (FDA) for diagnosing M.tuberculosis infection in Pulmonary TB but none of this assay is approved for diagnosing TBM. These assays offer widely variable sensitivities for diagnosing TBM and require costly indigenous platforms making them unaffordable for routine diagnosis of TBM in developing countries. Some of the commercial available assays are:

\subsection{Gen-probe amplified m. tuberculosis direct test (AMTD; gen-probe, CA)}


This assay amplifies target M. tuberculosis rRNA by transcription at a constant temperature and detects the amplicon by probing with chemiluminescence-labeled complementary DNA. A luminometer detects stable RNA-DNA hybrids. This test has shown sensitivity varying from 50 to $77 \%$ with specificity of $95-99 \%$.

\subsection{Xpert MTB/RIF (Cepheid, CA, USA)}

Recent introduction of the Gene Xpert System (Cepeid) has been a breakthrough for simultaneous detection of $\mathrm{M}$. tuberculosis and Rifampicin drug resistance. Thisis an automated rapid system which employs three specific primers and five unique molecular probes to detect MTB and Rifampicin resistance simultaneously in single-use sealed disposable cartridges by nested real time quantitative PCR. The assay takes less than 2 hour and requires minimal technical training to operate with minimal risk of contamination. The published Xpert threshold is approximately 100-130 CFU/ml of sample. The Xpert system has been evaluated in limited number of studies for diagnosing TBM and has shown sensitivity varying from $27-86 \%$ with specificity of $99-100 \%$ but is emerging as a potential diagnostic tool for timely TBM diagnosis and drug resistance.

\subsection{Line-probe assays (LPAs)}

LPA were primarily approved for identification of M. tuberculosis complex and rapid detection of drug susceptibility in M. tuberculosis isolated from culture. Later one of these assays (Genotype MTBDRplus) was approved by World Health Organization for direct detection of multi drug resistant $\mathrm{M}$.tuberculosis from smear positive/negative pulmonary specimens.

These assays are based on amplification of specific fragments of the M. tuberculosis genome (regions coding for drug resistance) followed by hybridization of PCR products to large number of oligonucleotide probes immobilized on membranes. Any nucleotide change in the target gene prevents the hybridization of the target and the corresponding wild-type probes and permits its hybridization to the corresponding mutation probe. The hybrids formed are subsequently detected calorimetrically. The presence of only wild type bands indicates that the strain is sensitive and absence of any wild type band with appearance of mutation bands indicates that strain is resistant. This assay also enables to identify the commonly occurring mutations responsible for drug resistance thus the molecular basis of resistance.

Two LPAs are currently available for the detection of drug susceptibility in M. tuberculosis:

\section{INNO-LiPA Rif. TB (Inn genetics, gent, Belgium)}

This assay is capable of identifyingM. tuberculosis complex along with Rifampicin drug resistance from the culture isolates.This assay has not been sufficiently evaluated for detection of drug resistance from TBM patients.

\section{Genotype MTBDRplus (Hain Lifescience GbmH, Neh- ren Germany)}

GenoType ${ }^{\circledR}$ MTBDRplus line probe assay (HAIN Life Sciences, Germany) was initially approved for detection of multi drug resistance from M. tuberculosis cultures. Later the test was approved by World Health Organization for direct detection of multi drug resistant M.tuberculosis from smear positive/negative pulmonary specimens. This assay is capable of detecting both low level and high level of INH resistance and Rifampicin resistance.

This test is useful in the rapid diagnosis of Drug-resistant tuberculous meningitis. This assay is used for diagnosing MDR tuberculosis from direct CSF sample and found as a rapid, simple and feasible alternative for diagnosing and detecting drug resistance from TBM patients.
Loop-mediated isothermal amplification (Eiken Chemical Co., Ltd, Tokyo, Japan)

LAMP technology is a simple, rapid, specific and cost-effective nucleic acid amplification method which does not require any sophisticated equipment or skilled personnel and is an attractive and feasible option in resource-limited settings. The assay is characterized by the use of 4 different primers specifically designed to recognize 6 distinct regions on the target gene and the reaction process proceeds at a constant temperature using strand displacement reaction. Amplification and detection of gene can be completed in a single step, by incubating the mixture of samples, primers, DNA polymerase with strand displacement activity and substrates at a constant temperature (about $65^{\circ} \mathrm{C}$ ). It provides high amplification efficiency, with DNA being amplified 1091010 times in 15-60 minutes. A large amount of product is formed, due to the auto displacement activity of the enzyme which can be detected by a color change of the reaction mixture in ambient light with a DNA binding dye.

A small study evaluating the use of LAMP on CSF for TBM diagnosis demonstrated good performance (sensitivity $88 \%$ and specificity $90 \%$ ) with better sensitivity than nested-PCR

\section{Indirect assays}

\subsection{Adenosine deaminase (ADA)}

Adenosine deaminase is produced by $\mathrm{T}$ lymphocytes and whose levels are elevated in patients whose CSF is infected with $\mathrm{M}$. tuberculosis. ADA is an important enzyme in purine metabolism, which irreversibly deaminates adenosine, converting it to inosine.ADA is found widely distributed in tissues and body fluids its presence is associated largely with lymphocytic proliferation and differentiation and is considered to be a marker of cell-mediated immunity.This is elevated in a variety of infections and disease involving the immune system and is an indirect predictor of CNS tuberculosis. Several studies have been performed to evaluate the utility of ADA measurements in the CSF to improve the diagnosis of TBMand measured sensitivities and sensitivities range from 44 to $100 \%$ [Thwaites et al, 2004, Kashyap et al, 2004, 2006]. CSF ADA level more than 5-15 iu/L adds as a corroborative evidence for diagnosing TBM. None of the cut-off values can be used to discriminate between TBM and bacterial meningitis. Moreover, high CSF ADA levels may also be seen in patients with malaria, lymphoma, pyogenic and cryptococcal meningitis and brucellosis. So, it is not recommended as a routine diagnostic test [Rock et al, 2008].

\subsection{Serology}

Numerous commercial serological tests are marketed in many parts of the world including India and used for diagnosis of TB as adjunct to microscopy and culture. ELISA test for antigen (usually A60 antigen) which is a serological test to detect specific antibody response in TB patients by using specific antigen. The antigen currently available are neither sufficiently specific nor sensitive and value of the test is similar to tuberculin test. ELISA test has a supportive value for the diagnosis of extrapulmonary TB.

Commercial serological tests provide inconsistent and imprecise findings resulting in highly variable values for sensitivity and specificity. Systematic reviews of serological tests for diagnosing extra pulmonary tuberculosis have demonstrated a highly variable sensitivity (0-100\%) and specificity (59-100\%) for the individual serological tests with a very low overall data quality and WHO strongly recommends that these tests should not be used for the diagnosing TB. There is no evidence that existing commercial serological assays improve patient outcomes, and high proportions of false positives and false negative results adversely impact patient safety. 


\subsection{Tuberculin skin test}

The TST has been used for the diagnosis of extra pulmonary tuberculosis; however, false-positive reactions occur as a result of previous BacilleCalmette-Gue'rin (BCG) vaccination or sensitization to NTM, and false-negative results occur in the immunocompromised patients, elderly persons or overt forms of TB. A positive tuberculin skin testing may provide some clue to the diagnosis and patient should further be investigated to establish the diagnosis of CNS TB.

\subsection{Tuberculostearic acid}

Tuberculostearic acid is a fatty acid component of the M. tuberculosis cell wall, which has been detected in CSF of patients with TBM via various forms of gas chromatography [French $\mathrm{G} \mathrm{L}$ et al, 1987]. Although this method has good sensitivity and specificity in limited studies, the requirement for expensive equipment and considerable expertise has limited the clinical use of this technique.

\subsection{Cerebrospinal fluid lactate levels}

CSF lactate levels have been used as a diagnostic marker for central nervous system infections. Lactate is produced by bacterial anaerobic metabolism, and increased CSF levels have been reported in patients with bacterial meningitis and TBM. A CSF lactate levels of 5-10 mmol/l support a diagnosis of TBM and high initial levels are associated with death. However, this marker has not been formally validated as a diagnostic test for TBM.

\subsection{Cytokine assays}

TB blood tests include interferon-gamma release assay which measures how the immune system reacts to the tubercular bacteria The measurement of Interferon-gamma release (IGRA) in whole blood or peripheral blood mononuclear cells in response to $\mathrm{M}$ .tuberculosis infection can serve as a corroborative tool for diagnosing TBM but there is no evidence to support the use of these assays to diagnose TBM.

Quantiferon-TB $\odot$ and T-Spot TB test are two such available assays but these does not differentiate between latent and active TB infection and have no utility in disease diagnosis and treatment in highly endemic countries.

QTB-G (Quantiferon TB Gold) can be applied on blood sample and is not affected by prior BCG vaccinationso greater sensitivity. The results are obtained within 3-4 days.

Combinations of CSF IGRA with other CSF tests such as ADA, PCR may improve the diagnosis of TBM [Thwaites et al 2009].

\section{Histo-pathological examination (Biopsy)}

Granulation tissue which is surgically accessible should be biopsied and provide a conclusive evidence of tubercular etiology. A tissue biopsy has much higher diagnostic yield than CSF for the diagnosis of tuberculoma and spinal tuberculosis. Eloquent areas of the brain which are not surgical accessible for open surgery, stereotactic biopsy is a better option. Some patients of brain and spinal tuberculosis may not respond to ATT and continue to deteriorateand neuroradiology also reveals persistence of the lesion. In such patients, a possibility of multi-drug resistant Tuberculosis (MDR TB) should be considered. Stereotactic biopsy for brain lesions and CT guided biopsy of granulation tissue of the paraspinal lesions are very useful for biopsy and culture \& sensitivity testing before starting second line ATT.

\section{Pragmatic approach to investigate a patient suspected to have CNS TB}

Due to various limitations, the diagnosis ofCNS tuberculosisis often presumptive and is based on clinical features, cerebrospinal fluid(CSF) findings, imaging findings and response to antituberculous drug therapy. Conventional methods like microscopy and cultureare considered gold-stanadard for the diagnosis of tuberculosis. However, it is not possible to get a positive result in all cases of CNS tuberculosis. AFB are less commonly found in the CSF of patients with cerebral tuberculoma or spinal tuberculosis and tissue examination is usually required to confirm the diagnosis. Thwaites $G$ et al have concluded that once the antituberculosis medication is commenced the sensitivity of smear and culture falls rapidly. The search for the AFB in CSF and tissue remains the most preferred diagnostic test for the CNS TB. However, practically the things are not that simple as once the antituberculosis medication is commenced the sensitivity of smear and culture falls rapidly. Thwaites and coworkers suggested in their research that the culture is too slow to help in initial treatment decision. There are various other tests like tubercular skin test, CSF adenosine deaminase, nucleic acid amplification (NAA) assay, IGRA and polymerase chain reaction (PCR) which also are part of diagnostic workup of CNS TB.However, their diagnostic accuracy are highly variable [Thwaites et al. 2004, 2009].

\section{Conclusion}

Early and accurate diagnosis is key to the success of CNS TB treatment. These investigations may provide an important clue to the diagnosis and treatment of a particular case. To conclude, there is no single test which can diagnose Neurotuberculosis. All the tests have their merits and limitations. So, a pragmatic approach is required to assess all the patients suspected to have CNS tuberculosis. FNAC from the enlarged lymph node should be done and it should also be used for AFB stain, PCR culture and sensitivity.Ziehl-Neelsen staining for acid-fast bacilli (AFB) is a quick and inexpensive method. Culture $\&$ drug susceptibility is gold standard, but culture results are available only after a few weeks and it is positive only in few cases. Drug susceptibility test (DST) is useful to start second line ATT. New and alternate methods should be used to diagnose CNS TB whenever the clinical presentation is subtle and clinical and routine laboratory investigations could not establish the diagnosis.

\section{References}

[1] Gautam VKS, Khurana S, Singh R (2013) Diagnostic and therapeutic challenges in the surgical management of CNS tuberculoma, International Journal of Medicine \& Health Sciences, April 2013 Vol-2; Issue-2, 161-169.

[2] Malik Z I, Ishtiaq O, Shah N H, Anwer F \&Baqai H Z ( 2002) Analysis and outcome of 30 patients with Tuberculous Meningitis.Pakistan Journal of Medical Research; Vol.41 No.4, 137-41.

[3] Naughten E, Weindling A M, Newton R \&. Bower B D (1981) Tuberculous meningitis in children. Recent experience in two English centres. Lancet. Oct, 31; 2 (8253):973975.http://dx.doi.org/10.1016/s0140-6736(81)91166-1.

[4] Kent S J, Crowe S M, Yung A, Lucas C R \&MijchA M (1993) Tuberculous meningitis: a 30-year review. Clinical Infectious Diseases. 17:987-994. http://dx.doi.org/10.1093/clinids/17.6.987.

[5] Verdon R, Chevret S, Laissy J P \& Wolff M (1996). Tuberculous meningitis in adults: review of 48 cases. Clinical Infectious Diseases. 22:982-988.http://dx.doi.org/10.1093/clinids/22.6.982.

[6] Thwaites G E, Chau T T\& Farrar J J (2004). Improving the bacteriological diagnosis of tuberculous meningitis. Journal of Clinical Microbiology. 379.http://dx.doi.org/10.1128/JCM.42.1.378-379.2004.

[7] Thwaites G E, Caws M, Chau T T, Dung N T, Campbell J I, Phu N H,Hien T T, White N J \& Farrar J J (2004). Comparison of conventional bacteriology with nucleic acid amplification (amplified mycobacterium direct test) for diagnosis of tuberculous meningitis be- 
fore and after inception of antituberculosis chemotherapy. Journal of Clinical Microbiology. 42: 9961002.http://dx.doi.org/10.1128/JCM.42.3.996-1002.2004.

[8] Jeren, T \&Beus I (1982). Characteristics of cerebrospinal fluid in tuberculous meningitis. ActaCytologica. 26:678-680.

[9] Sutlas P N, Unal A, Forta H, Senol S, \&Kirbas D (2003). Tuberculous meningitis in adults: review of 61 cases. Infection 31:387-391.

[10] Updated guidelines for the use of Nucleic acid amplification tests in the diagnosis of tuberculosis, http://www.cdc.gov/tb MMWR weekly January 16, 2009/58(01); 7-10.

[11] Cherian A, Thomas SV. Central nervous system tuberculosis. African Health Sciences Vol 11, No.1, March 2011, 116-127.

[12] Kashyap R S, Kainthla R P, Satpute R M, Agarwal N P, Chandak N H, Purohit H J, Taori G M \&Daginawala H F (2004). Differential diagnosis of tuberculous meningitis from partially-treated pyogenic meningitis by cell ELISA. BMC Neurology. 4:16.http://dx.doi.org/10.1186/1471-2377-4-16.

[13] Kashyap R S, Kainthla R P, Mudaliar A V,Purohit H J, Taori G M, \&Daginawala H F (2006). Cerebrospinal fluid adenosine deaminase activity: a complementary tool in the early diagnosis of tuberculous meningitis. Cerebrospinal Fluid Research. March 30, 3: 5.

[14] Rock RB, Olin M, Baker CA, Molitor TW\& Peterson PK (2008). Central nervous system tuberculosis: pathogenesis and clinical aspects. Clinical Microbiology Reviews.Apr; 21(2):24361http://dx.doi.org/10.1128/CMR.00042-07.

[15] French G L, Teoh R, Chan C Y, Humphries M J, Cheung S W \&O'Mahony G (1987) Diagnosis of tuberculous meningitis by detection oftuberculostearic acid in cerebrospinal fluid. Lancet.July 18; 2(8551):117-119.

[16] Thwaites G, Fisher M, Hemingway C, Scott G, Solomon T, Innes J British infection society guidelines for the diagnosis and treatment of tuberculosis of the central nervous system in adults and children. Journal of Infection (2009), 59, 167 187.http://dx.doi.org/10.1016/j.jinf.2009.06.011. 